\title{
A checklist of the spiders (Arachnida, Araneae) of the Polokwane Nature Reserve, Limpopo Province, South Africa
}

\author{
SUSAN M. DIPPENAAR \\ ${ }^{1}$ Department of Biodiversity \\ School of Molecular E Life Sciences \\ University of Limpopo \\ South Africa
}

ANSIE S. DIPPENAAR-SCHOEMAN

ARC-Plant Protection Research Institute South Africa

\author{
MOKGADI A. MODIBA ${ }^{1}$ \\ THEMBILE T. KHOZA ${ }^{1}$
}

Correspondence to: Susan M. Dippenaar

e-mail: susand@ul.ac.za

Postal Address: Private Bag X1106, Sovenga 0727, Republic of South Africa

\begin{abstract}
As part of the South African National Survey of Arachnida (SANSA), spiders were collected from all the field layers in the Polokwane Nature Reserve (Limpopo Province, South Africa) over a period of a year (2005-2006) using four collecting methods. Six habitat types were sampled: Acacia tortillis open savanna; A. rehmanniana woodland, false grassland, riverine and sweet thorn thicket, granite outcrop; and Aloe marlothii thicket. A total of 13821 spiders were collected (using sweep netting, tree beating, active searching and pitfall trapping) represented by 39 families, 156 determined genera and 275 species. The most diverse families are the Thomisidae (42 spp.), Araneidae (39 spp.) and Salticidae (29 spp.). A total of 84 spp. (30.5\%) were web builders and 191 spp. (69.5\%) wanderers. In the Polokwane Nature Reserve, $13.75 \%$ of South African species are presently protected.
\end{abstract}

Keywords: Arachnida, Araneae, diversity, habitats, conservation

In the early 1990s, South Africa was recognised, in terrestrial terms, as a biologically very rich country and even identified as the world's 'hottest hotspot' (Myers 1990). After the United Nations Conference on Environment and Development in 1992, South Africa ratified the Convention on Biological Diversity in 1995 with the main goal to develop a strategic plan for conservation and sustainable use of biodiversity. One of the national efforts identified as necessary to accomplish the goal was to discover, describe and to make an inventory of the species of South Africa. As a result of this, the South African National Survey of Arachnida (SANSA) was established in 1997 with the aim to develop an inventory of the arachnofauna of South Africa (Dippenaar-Schoeman \& Craemer 2000). Arachnids are well represented in South Africa, with nine of the twelve arachnid orders occurring here. However, even though they constitute an abundant and successful group, they are still poorly sampled with little existing knowledge about their diversity and distribution within certain ecoregions.

South Africa has a very rich spider fauna represented by 69 families, 469 genera and about 2000 species that occur in all of the ecoregions of South Africa (Dippenaar-Schoeman 2002a). Unfortunately, the lack of taxonomic expertise makes it impossible to identify some spider families to species and even generic levels, which could lead to under-appreciation and estimation of the actual species pool and undermines meaningful conservation. As part of SANSA, this study addressed specifically the objective to compile species lists within the Savanna Biome, starting with checklists of the conserved areas where conservation strategies are already in place.

The Savanna Biome is one of the world's major biomes and covers about one third of South Africa (Low \& Rebelo 1996). It is especially well developed in the Kalahari, parts of Limpopo and KwaZulu-Natal, Mpumalanga and the Eastern Cape. Savanna is characterised by a grassy ground layer and a distinct upper layer of woody plants that can be identified as shrubveld, woodland or bushveld. The delimiting factor is complex for the Savanna Biome and includes mainly rainfall (235 to 1000 $\mathrm{mm}$ per year) and/or frost (0-120 days/year). Reports on the spiders of the Savanna Biome in South Africa are restricted to that of Roodeplaat Dam Nature Reserve (Dippenaar-Schoeman et al. 1989), Makalali Game Reserve (Whitmore et al. 2001, 2002), Western Soutpansberg (Foord et al. 2002), Kruger National Park (Dippenaar-Schoeman \& Leroy 2003), Sovenga Hill (Modiba et al. 2005) and Ndumo Game Reserve (Haddad et al. 2006).

This is the second survey of arachnids from the Polokwane region, Limpopo Province. The study was undertaken in the Polokwane Nature Reserve (PNR) which lies in the Savanna Biome, and is situated in the Central Bushveld Bioregion, specifically the Polokwane Plateau Bushveld vegetation unit (Mucina et al. 2005, Mucina \& Rutherford 2006). A significant portion of the reserve includes a unique and threatened habitat type known as the Pietersburg Plateau False Grassland. The reserve was given conservation status and was proclaimed as a municipal reserve after officially being opened in 1971 . Currently the reserve conserves one of the largest pristine examples of this habitat type, along with its associated plant and animal species.

As part of the MSc studies of the third and fourth authors, spiders were sampled from six habitat types over a period of a year using four collecting methods, sampling all the field layers. This paper is the first in a series of papers on the reserve and provides only an annotated checklist for the spiders of the reserve. Detailed information about the abundance and species richness of spiders collected from different habitat types, during different seasons and using different sampling methods will be published in succeeding papers. 


\section{STUDY AREA}

The Polokwane Nature Reserve $\left(23^{\circ} 58^{\prime} \mathrm{S} 29^{\circ} 28^{\prime} \mathrm{E}\right)$ covers an area of approximately 3200 ha and lies on an elevated plateau with an altitude ranging between 1200 and $1500 \mathrm{~m}$ above sea level. The climate is moderate with mean summer day-time high of $28^{\circ} \mathrm{C}$ and a mean winter night-time low of $5{ }^{\circ} \mathrm{C}$. The average annual rainfall for the area is $478 \mathrm{~mm}$. The Polokwane Plateau Bushveld vegetation unit is characterised by open Themeda grassland with scattered Acacia trees and bush clumps. The dominant trees are Acacia tortillis (Forssk.) Hayne and A. rehmanniana Schinz. Other interesting plant communities and habitats in the reserve include riverine and sweet thorn thickets, granite outcrops, quartzite pebble slopes, saline patches and a mountain aloe, Aloe marlothii A. Berger thicket situated on a shale outcrop (Grosel pers. comm.).

\section{METHODS}

Six different habitat types were selected subjectively to represent most of the habitat diversity in the reserve and included: 1. Acacia tortillis open savanna, 2. A. rehmanniana woodland, 3. false grassland, 4 . riverine and sweet thorn thicket, 5. a northern slope granite outcrop, and 6. Aloe marlothii thicket on shale outcrop.

Four collecting methods were used, namely grass sweep netting, tree beating, active searching and pitfall trapping. Leaf litter sifting was attempted once on the A. rehmanniana woodland site but was discontinued because of insufficient leaf litter. Sampling was conducted once a month in each of the six habitat types for a duration of 12 months (March 2005 to February 2006).

Pitfall trapping: Containers with a $10 \mathrm{~cm}$ diameter were planted with the upper rim level with the ground surface and covered by a funnel leading to a smaller container filled with $70 \% \mathrm{EtOH}$ to immobilise and preserve caught specimens. Ten traps, $10 \mathrm{~m}$ apart, were planted per habitat site, consisting of a central trap with three arms in three different directions, each arm consisting of three traps. The traps were checked every day during the week of sampling per site. The pitfall traps were open for five consecutive days, resulting in 60 trap days per site and therefore 360 trap days for all sampling sites during the sampling period of one year. This resulted in a total of 3600 pitfall trap samples taken during the study.

Sweep netting: A sweep net with a diameter of $31 \mathrm{~cm}$ was used to sample through grass and herbs. Sweeping was conducted once a month for two hours at each site, while walking from the central pitfall trap throughout the sampling site. Collected material was gathered into marked plastic bags and sorted in the laboratory.

Tree beating: Trees and shrubs were beaten with a $0.21 \mathrm{~kg}$ baton to dislodge specimens which were caught on a sheet and collected in plastic bags. Bigger trees received up to 20 beatings while smaller trees and shrubs received fewer beatings. This method was applied once a month for two hours at each site. Collected material was sorted in the laboratory.

Active searching was undertaken once a month for two hours at each site. The area covered was from the central pitfall trap working outwards and covered the whole sampling site. Specimens were searched and collected from all field layers.

Collected spiders were fixed and preserved in $70 \% \mathrm{EtOH}$ and identified up to family level using the African spider family keys (Dippenaar-Schoeman \& Jocqué 1997) while genus and species determinations were done by the second author. Voucher specimens were deposited in the National Collection of Arachnida (NCA) at the Plant Protection Research Institute in Pretoria, an institute of the Agricultural Research Council.
Two main guilds can be distinguished among spiders, namely wanderers and web builders. The wanderers can be further divided into plant wanderers (PW) and ground wanderers (GW). Plant wanderers were separated and placed in a guild based on the vegetation type that they were most commonly found on, namely plant wanderers found on foliage (PWF), plant wanderers found on grass (PWG) and plant wanderers found on bark (PWB) while ground wanderers may be free living or burrow dwelling (BD). The web-building spiders can also be subdivided into different guilds based on the structure of the webs that they build: sheet-web builders (SWB), spaceweb builders (SPWB), orb-web builders (OWB), funnel-web builders (FWB), retreat-web builders (RWB), tube-web builders (TWB) and gumfoot-web builders (GWB).

\section{RESULTS AND DISCUSSION}

A total of 13821 spiders were caught during the sampling period, representing 39 families, 156 determined genera and 275 species (see Table 1). The 39 families represent $56.5 \%$ of the currently recognised families for South Africa (DippenaarSchoeman \& Jocqué 1997). The representation and numbers caught (irrespective of other factors such as length of sampling period, size of sampling area etc.) compare favourably with

TABLE 1

Spider families collected in the Polokwane Nature Reserve indicating the number of species and percentage of the total number of specimens collected

\begin{tabular}{|c|c|c|c|c|}
\hline FAMILIES & GENERA & SPECIES & SPECIMENS & $\%$ \\
\hline Agelenidae & 2 & 2 & 11 & 0.08 \\
\hline Amaurobiidae & 1 & 1 & 1 & 0.01 \\
\hline Ammoxenidae & 1 & 1 & 61 & 0.44 \\
\hline Araneidae & 23 & 39 & 916 & 6.63 \\
\hline Barychelidae & 1 & 1 & 3 & 0.02 \\
\hline Caponiidae & 1 & 1 & 16 & 0.12 \\
\hline Clubionidae & 1 & 2 & 17 & 0.12 \\
\hline Corinnidae & 4 & 4 & 7 & 0.05 \\
\hline Ctenidae & 1 & 1 & 3 & 0.02 \\
\hline Cyrtaucheniidae & 1 & 1 & 6 & 0.04 \\
\hline Dictynidae & 1 & 1 & 5 & 0.04 \\
\hline Eresidae & 3 & 3 & 2547 & 18.43 \\
\hline Gnaphosidae & 13 & 25 & 499 & 3.61 \\
\hline Hersiliidae & 1 & 1 & 11 & 0.08 \\
\hline Idiopidae & 3 & 3 & 7 & 0.05 \\
\hline Linyphiidae & 4 & 4 & 5 & 0.04 \\
\hline Lycosidae & 8 & 21 & 784 & 5.67 \\
\hline Mimetidae & 1 & 1 & 4 & 0.03 \\
\hline Miturgidae & 1 & 3 & 289 & 2.09 \\
\hline Nemesiidae & 1 & 1 & 2 & 0.01 \\
\hline Nephilidae & 1 & 1 & 31 & 0.22 \\
\hline Oonopidae & 1 & 1 & 1 & 0.01 \\
\hline Oxyopidae & 3 & 16 & 3415 & 24.71 \\
\hline Palpimanidae & 2 & 3 & 53 & 0.38 \\
\hline Philodromidae & 7 & 12 & 621 & 4.49 \\
\hline Pholcidae & 1 & 1 & 4 & 0.03 \\
\hline Pisauridae & 5 & 6 & 232 & 1.68 \\
\hline Prodidomidae & 1 & 1 & 9 & 0.07 \\
\hline Salticidae & 18 & 29 & 1056 & 7.64 \\
\hline Scytodidae & 1 & 1 & 2 & 0.01 \\
\hline Segestriidae & 1 & 1 & 4 & 0.03 \\
\hline Selenopidae & 2 & 2 & 9 & 0.07 \\
\hline Sparassidae & 3 & 5 & 25 & 0.18 \\
\hline Tetragnathidae & 1 & 2 & 4 & 0.03 \\
\hline Theraphosidae & 3 & 3 & 10 & 0.07 \\
\hline Theridiidae & 9 & 22 & 778 & 5.63 \\
\hline Thomisidae & 17 & 42 & 2212 & 16.00 \\
\hline Uloboridae & 2 & 4 & 42 & 0.30 \\
\hline Zodariidae & 6 & 7 & 119 & 0.86 \\
\hline TOTAL & 156 & 275 & 13821 & 100 \\
\hline
\end{tabular}

(*value excluding undetermined genera) 
TABLE 2

Checklist of the spiders of the Polokwane Nature Reserve, Limpopo Province, South Africa (PW: plant wanderer; GW: ground wanderer; BD: burrow dweller; SWB: sheet-web builder; SPWB: space-web builder; OWB: orb-web builder; FWB: funnel-web builder; RWB: retreat-web builder; GWB: gumfoot-web builder; TWB: tube-web builder)

* possibly new

\begin{tabular}{|c|c|c|c|}
\hline FAMILY & SPECIES & GUILD & FIELD TYPE \\
\hline \multirow[t]{2}{*}{ Agelenidae } & Agelena sp. (immature) & FWB & on soil \\
\hline & Olorunia sp. (Immature) & FWB & on soil \\
\hline Amaurobiidae & Pseudauximus sp. (immature) & RWB & debri \\
\hline Ammoxenidae & Ammoxenus amphalodes Dippenaar \& Meyer, 1980 & GW & soil \\
\hline \multirow[t]{39}{*}{ Araneidae } & Acanthepeira sp. * & OWB & vegetation \\
\hline & Araneidae $s p$. (genus undetermined) & OWB & vegetation \\
\hline & Araneus apricus (Karsch, 1884) & OWB & vegetation \\
\hline & Araneus coccinella Pocock, 1898 & OWB & vegetation \\
\hline & Araneus nigroquadratus Lawrence, 1937 & OWB & vegetation \\
\hline & Araniella sp. $1^{*}$ & OWB & vegetation \\
\hline & Araniella sp. $2^{*}$ & OWB & vegetation \\
\hline & Araniella sp. $3^{*}$ & OWB & vegetation \\
\hline & Argiope aurocincta Pocock, 1898 & OWB & vegetation \\
\hline & Argiope australis (Walckenaer, 1805) & OWB & vegetation \\
\hline & Argiope lobata (Pallas, 1772) & OWB & vegetation \\
\hline & Argiope trifasciata (Forskal, 1775) & OWB & vegetation \\
\hline & Argiope sp. (immature) & OWB & vegetation \\
\hline & Caerostris sexcuspidata (Fabricius, 1793) & OWB & vegetation \\
\hline & Chorizopes sp. * & OWB & vegetation \\
\hline & Cyclosa insulana (Costa, 1834) & OWB & vegetation \\
\hline & Cyphalanothus larvatus (Simon, 1881) & OWB & vegetation \\
\hline & Cyrtophora citricola (Forskal, 1775) & OWB & vegetation \\
\hline & Gasteracantha sanguinolenta C.L. Koch, 1884 & OWB & vegetation \\
\hline & Gea infuscata Tullgren, 1910 & OWB & vegetation \\
\hline & Hypsosinga lithyphantoides Caporiacco, 1947 & OWB & vegetation \\
\hline & Hypsosinga sp. 2 & OWB & vegetation \\
\hline & Larinia natalensis (Grasshoff, 1971) & OWB & vegetation \\
\hline & Lipocrea longissima (Simon, 1881) & OWB & vegetation \\
\hline & Mahembea hewitti (Lessert, 1930) & OWB & vegetation \\
\hline & Nemoscolus elongatus Lawrence, 1947 & OWB & vegetation \\
\hline & Nemoscolus sp. 2 & OWB & vegetation \\
\hline & Neoscona blondeli (Simon, 1886) & OWB & vegetation \\
\hline & Neoscona moreli (Vinson, 1863) & OWB & vegetation \\
\hline & Neoscona penicillipes (Karsch, 1879) & OWB & vegetation \\
\hline & Neoscona quincasea Roberts, 1983 & OWB & vegetation \\
\hline & Neoscona subfusca (C.L. Koch, 1837) & OWB & vegetation \\
\hline & Paraplectana sp. 1 & OWB & vegetation \\
\hline & Pararaneus cyrtoscapus (Pocock, 1898) & OWB & vegetation \\
\hline & Prasonica sp. $1^{*}$ & OWB & vegetation \\
\hline & Pycnacantha tribulus (Fabricius, 1781) & OWB & vegetation \\
\hline & Singa lawrencei (Lessert, 1930) & OWB & vegetation \\
\hline & Singa sp. 2 & OWB & vegetation \\
\hline & Singa sp. 3 & OWB & vegetation \\
\hline Caponiidae & Caponia chelifera Lessert, 1936 & GW & soil \\
\hline \multirow[t]{2}{*}{ Clubionidae } & Clubiona abbajensis Strand, 1906 & PW & vegetation \\
\hline & Clubiona sp. (immature) & PW & vegetation \\
\hline \multirow[t]{4}{*}{ Corinnidae } & Casteineira sp. 1 & GW & soil \\
\hline & Cetonana simoni (Lawrence, 1942) & GW & soil \\
\hline & Copa flavoplumosa Simon, 1885 & GW & soil \\
\hline & Graptartia mutillica Haddad, 2004 & GW & soil \\
\hline Ctenidae & Ctenus sp. 1 & GW & soil \\
\hline Cyrtaucheniidae & Ancylotrypa brevipalpis (Hewitt, 1916) & $\mathrm{BD}$ & burrow soil \\
\hline Dictynidae & Archaeodictyna sp. 1 & RWB & vegetation \\
\hline \multirow[t]{3}{*}{ Eresidae } & Dresserus colsoni Tucker, 1920 & RWB & soil debri \\
\hline & Gandanameno fumosus (C.L. Koch, 1837) & RWB & tree \\
\hline & Stegodyphus dumicola Pocock, 1898 & RWB & tree \\
\hline
\end{tabular}


Table 2 continued . . .

\begin{tabular}{|c|c|c|c|}
\hline FAMILY & SPECIES & GUILD & FIELD TYPE \\
\hline \multirow[t]{25}{*}{ Gnaphosidae } & Aneplasa sp. 1 & & \\
\hline & Asemesthes ceresicola Tucker, 1923 & GW & soil \\
\hline & Asemesthes decoratus Purcell, 1908 & GW & soil \\
\hline & Camillina aestus Tucker, 1923 & GW & soil \\
\hline & Camillina maun Platnick \& Murphy, 1987 & GW & soil \\
\hline & Camillina procurva (Purcell, 1908) & GW & soil \\
\hline & Drassodes bechuanicus Tucker, 1923 & GW & soil \\
\hline & Drassodes solitarius Purcell, 1907 & GW & soil \\
\hline & Drassodes splendens Tucker, 1923 & GW & soil \\
\hline & Drassodes stationis Tucker, 1923 & GW & soil \\
\hline & Echemus sp. 1 & GW & soil \\
\hline & Poecilochoa sp. 1 & GW & soil \\
\hline & Scotophaeus marleyi Tucker, 1923 & GW & soil \\
\hline & Setaphis anchoralis Purcell, 1908 & GW & soil \\
\hline & Setaphis arcus Tucker, 1923 & GW & soil \\
\hline & Setaphis subtilis (Simon, 1897) & GW & soil \\
\hline & Trachyzelotes jaxartensis (Kroneberg, 1875) & GW & soil \\
\hline & Trephopoda hanoveria Tucker, 1923 & GW & soil \\
\hline & Upognampa parvipalpa Tucker, 1923 & GW & soil \\
\hline & Xerophaeus appendiculatus Purcell, 1907 & GW & soil \\
\hline & Xerophaeus bicavus Tucker, 1923 & GW & soil \\
\hline & Zelotes reduncus (Purcell, 1907) & GW & soil \\
\hline & Zelotes tuckeri Roewer, 1951 & GW & soil \\
\hline & Zelotes ungulus Tucker, 1923 & GW & soil \\
\hline & Zelotes sp. 4 & GW & soil \\
\hline Hersiliidae & Tyrotama soutpansbergensis Foord \& Dippenaar-Schoeman, 2005 & RWB & soil \\
\hline \multirow[t]{3}{*}{ Idiopidae } & Ctenolophus fernoulheti Hewitt, 1913 & $\mathrm{BD}$ & burrow soil \\
\hline & Idiops monticola (Hewitt, 1916) & $\mathrm{BD}$ & burrow soil \\
\hline & Idiops sp. 1 & $\mathrm{BD}$ & burrow soil \\
\hline \multirow[t]{4}{*}{ Linyphiidae } & Pelecopsis sp. 1 & SWB & vegetation \\
\hline & Meioneta sp. 1 & SWB & vegetation \\
\hline & Mecynidis sp. 1 & SWB & vegetation \\
\hline & Microlinyphia sterilis (Pavesi, 1883) & SWB & vegetation \\
\hline \multirow[t]{21}{*}{ Lycosidae } & Evippomma squamulatum (Simon, 1898) & GW & soil \\
\hline & Geolycosa sp. 1 & GW & soil \\
\hline & Lycosa sp. 1 & GW & soil \\
\hline & Lycosa sp. 2 & GW & soil \\
\hline & Hogna sp. 1 & GW & soil \\
\hline & Hogna sp. 2 & GW & soil \\
\hline & Lycosidae sp. 1 (genus undetermined) & GW & soil \\
\hline & Lycosidae sp. 2 (genus undetermined) & GW & soil \\
\hline & Lycosidae sp. 3 (genus undetermined) & GW & soil \\
\hline & Lycosidae sp. 4 (genus undetermined) & GW & soil \\
\hline & Lycosidae sp. 5 (genus undetermined) & GW & soil \\
\hline & Lycosidae sp. 6 (genus undetermined) & GW & soil \\
\hline & Lycosidae sp. 7 (genus undetermined) & GW & soil \\
\hline & Pardosa leipoldti Purcell, 1903 & GW & soil \\
\hline & Pardosa sp. 2 & GW & soil \\
\hline & Pardosa sp. 3 & GW & soil \\
\hline & Pardosa sp. 4 & GW & soil \\
\hline & Proevippa wanlessi (Russell-Smith, 1981) & GW & soil \\
\hline & Proevippa sp. 2 & GW & soil \\
\hline & Trabea purcelli Roewer, 1951 & GW & soil \\
\hline & Zenonina albocaudata Lawrence, 1952 & GW & soil \\
\hline Mimetidae & Ero sp. $1^{*}$ & PW & vegetation \\
\hline \multirow[t]{3}{*}{ Miturgidae } & Cheiracanthium africanum Lessert, 1921 & PW & vegetation \\
\hline & Cheiracanthium furculatum Karsch, 1879 & PW & vegetation \\
\hline & Cheiracanthium vansoni Lawrence, 1936 & PW & vegetation \\
\hline Nemesiidae & Hermacha mazoena Hewitt, 1915 & $\mathrm{BD}$ & burrow soil \\
\hline
\end{tabular}


Table 2 continued . . .

\begin{tabular}{|c|c|c|c|}
\hline FAMILY & SPECIES & GUILD & FIELD TYPE \\
\hline Nephilidae & Nephila senegalensis (Walckenaer, 1842) & OWB & vegetation \\
\hline Oonopidae & Gamasomorpha humicola Lawrence, 1947 & GW & soil \\
\hline \multirow[t]{16}{*}{ Oxyopidae } & Hamataliwa fronticornis (Lessert, 1927) & PW & grass \\
\hline & Hamataliwa kulczynskii (Lessert, 1915) & PW & grass \\
\hline & Hamataliwa strandi Caporriacco, 1939 & PW & grass \\
\hline & Oxyopes affinis Lessert, 1915 & PW & grass \\
\hline & Oxyopes bedoti Lessert, 1915 & PW & grass \\
\hline & Oxyopes bothai Lessert, 1915 & PW & grass \\
\hline & Oxyopes hoggi Lessert, 1915 & PW & grass \\
\hline & Oxyopes jacksoni Lessert, 1915 & PW & grass \\
\hline & Oxyopes pallidecoloratus Strand, 1906 & PW & grass \\
\hline & Oxyopes russoi Caporiacco, 1940 & PW & grass \\
\hline & Oxyopes schenkeli Lessert, 1927 & PW & grass \\
\hline & Oxyopes tuberculatus Lessert, 1915 & PW & grass \\
\hline & Oxyopes sp. 10 & PW & grass \\
\hline & Oxyopes sp. 11 & PW & grass \\
\hline & Oxyopes sp. 12 & PW & grass \\
\hline & Peucetia viridis (Blackwall, 1858) & PW & grass \\
\hline \multirow[t]{3}{*}{ Palpimanidae } & Diaphorocellus biplagiatus Simon, 1893 & GW & soil \\
\hline & Palpimanus armatus Pocock, 1898 & GW & soil \\
\hline & Palpimanus transvaalicus Simon, 1893 & GW & soil \\
\hline \multirow[t]{12}{*}{ Philodromidae } & Ebo sp. 1* & PW & grass \\
\hline & Gephyrota sp. $1^{*}$ & PW & grass \\
\hline & Hirriusa variegata (Simon, 1895) & PW & grass \\
\hline & Philodromus browningi Lawrence, 1952 & PW & grass \\
\hline & Philodromus grosi Lessert, 1943 & PW & grass \\
\hline & Philodromus guineensis Millot, 1942 & PW & grass \\
\hline & Suemus punctatus Lawrence, 1938 & PW & grass \\
\hline & Thanatus dorsilineatus Jézéquel, 1964 & PW & grass \\
\hline & Thanatus sp. 2 & PW & grass \\
\hline & Tibellus gerhardi Van den Berg \& Dippenaar-Schoeman, 1994 & PW & grass \\
\hline & Tibellus hollidayi Lawrence, 1952 & PW & grass \\
\hline & Tibellus minor Lessert, 1919 & PW & grass \\
\hline Pholcidae & Smeringopus atomarius Simon, 1910 & SPWB & soil \\
\hline \multirow[t]{6}{*}{ Pisauridae } & Afropisaura sp. 1 & PW & vegetation \\
\hline & Euprosthenops australis Simon, 1898 & FWB & vegetation \\
\hline & Euprosthenopsis vuattouxi Blandin, 1977 & FWB & vegetation \\
\hline & Maypacius bilineatus (Pavesi, 1895) & PW & vegetation \\
\hline & Maypacius stuhlmanni (Bösenberg \& Lenz, 1895) & PW & vegetation \\
\hline & Rothus purpurissatus Simon, 1898 & PW & vegetation \\
\hline Prodidomidae & Theuma parva Purcell, 1907 & GW & soil \\
\hline \multirow[t]{17}{*}{ Salticidae } & Aelurillus sp. $1^{*}$ & GW & soil \\
\hline & Baryphas ahenus Simon, 1902 & PW & vegetation \\
\hline & Brancus bevisi Lessert, 1925 & PW & vegetation \\
\hline & Cosmophasis sp. 1 & GW & soil \\
\hline & Dendryphantes sp. 1 & PW & vegetation \\
\hline & Dendryphantes sp. 2 & PW & vegetation \\
\hline & Euophrys sp. 1 & PW & vegetation \\
\hline & Heliophanus debilis Simon, 1901 & PW & vegetation \\
\hline & Heliophanus demonstrativus Wesolowska, 1986 & PW & vegetation \\
\hline & Heliophanus insperatus Wesolowska, 1986 & PW & vegetation \\
\hline & Heliophanus transvaalicus Simon, 1901 & PW & vegetation \\
\hline & Hyllus treleaveni Peckham \& Peckham, 1902 & PW & vegetation \\
\hline & Hyllus sp. & PW & vegetation \\
\hline & Hyllus sp. 3 & PW & vegetation \\
\hline & Hyllus sp. 4 & PW & vegetation \\
\hline & Hyllus sp. 5 & PW & vegetation \\
\hline & Langelurillus sp. 1 & GW & soil \\
\hline
\end{tabular}


Table 2 continued . .

\begin{tabular}{|c|c|c|c|}
\hline FAMILY & SPECIES & GUILD & FIELD TYPE \\
\hline & Mogrus sp. 1 & PW & vegetation \\
\hline & Natta horizontalis Karsch, 1879 & GW & soil \\
\hline & Pellenes sp. 1 & PW & vegetation \\
\hline & Phlegra sp. 1 & GW & soil \\
\hline & Pseudicius sp. 1 & PW & vegetation \\
\hline & Rhene machadoi Berland \& Millot, 1941 & PW & vegetation \\
\hline & Stenaelurillus nigricaudus Simon, 1886 & GW & soil \\
\hline & Stenaelurillus $s p .1$ & GW & soil \\
\hline & Stenaelurillus sp. 2 & GW & soil \\
\hline & Stenaelurillus sp. 3 & GW & soil \\
\hline & Thyene inflata (Gerstäcker, 1873) & PW & vegetation \\
\hline & Thyenula aurantiaca (Simon, 1902) & PW & vegetation \\
\hline Scytodidae & Scytodes sp. 1 & GW & soil \\
\hline Segestriidae & Ariadna sp. 1 & TWB & soil \\
\hline \multirow[t]{2}{*}{ Selenopidae } & Anyphops sp. 1 & PW & tree \\
\hline & Selenops sp. 1 & PW & tree \\
\hline \multirow[t]{5}{*}{ Sparassidae } & Olios sp. 1 & PW & vegetation \\
\hline & Olios sp. 2 & PW & vegetation \\
\hline & Olios sp. 3 & PW & vegetation \\
\hline & Palystes superciliosus L. Koch, 1875 & PW & vegetation \\
\hline & Pseudomicrommata longipes (Bösenberg \& Lenz, 1895) & PW & vegetation \\
\hline \multirow[t]{2}{*}{ Tetragnathidae } & Leucauge decorata (Blackwall, 1864) & OWB & vegetation \\
\hline & Leucauge festiva (Blackwall, 1866) & OWB & vegetation \\
\hline \multirow[t]{4}{*}{ Theraphosidae } & Augacephalus junodi (Simon, 1904) & $\mathrm{BD}$ & burrow soil \\
\hline & Brachionopus pretoriae Purcell, 1904 & BD & burrow soil \\
\hline & Harpactira sp. 1 & BD & burrow soil \\
\hline & Harpactirella sp. 1 & BD & burrow soil \\
\hline \multirow[t]{22}{*}{ Theridiidae } & Argyrodes convivans Lawrence, 1937 & GWB & webs other spiders \\
\hline & Argyrodes zonatus (Walckenaer, 1842) & GWB & webs other spiders \\
\hline & Dipoena sp. 1 & GWB & vegetation \\
\hline & Enoplognatha molesta O.P.-Cambridge, 1904 & GWB & vegetation \\
\hline & Latrodectus geometricus C.L.Koch, 1841 & GWB & vegetation \\
\hline & Latrodectus renivulvatus Dahl, 1902 & GWB & vegetation \\
\hline & Phoroncidia eburnea (Simon, 1895) & GWB & vegetation \\
\hline & Steatoda sp. 1 & GWB & soil \\
\hline & Theridiidae $s p .1$ (genus undetermined) & GWB & vegetation \\
\hline & Theridiidae sp. 2 (genus undetermined) & GWB & Vegetation \\
\hline & Theridiidae sp. 3 (genus undetermined) & GWB & vegetation \\
\hline & Theridiidae sp. 4 (genus undetermined) & GWB & vegetation \\
\hline & Theridiidae sp. 5 (genus undetermined) & GWB & vegetation \\
\hline & Theridion purcelli O.P.-Cambridge, 1904 & GWB & vegetation \\
\hline & Theridion $s p .2$ & GWB & vegetation \\
\hline & Theridion sp. 3 & GWB & vegetation \\
\hline & Theridion sp. 4 & GWB & vegetation \\
\hline & Theridion sp. 5 & GWB & vegetation \\
\hline & Theridion sp. 6 & GWB & vegetation \\
\hline & Theridion sp. 7 & GWB & vegetation \\
\hline & Theridion sp. 8 & GWB & vegetation \\
\hline & Tidarren sp. 1 & GWB & vegetation \\
\hline \multirow[t]{10}{*}{ Thomisidae } & Avelis hystriculus Simon, 1895 & PW & vegetation \\
\hline & Camaricus nigrotesselatus Simon, 1895 & PW & vegetation \\
\hline & Heriaeus crassispinus Lawrence, 1942 & PW & grass \\
\hline & Heriaeus transvaalicus Simon, 1895 & PW & grass \\
\hline & Hewittia gracilis Lessert, 1928 & PW & grass \\
\hline & Misumenops rubrodecoratus Millot, 1942 & PW & grass \\
\hline & Monaeses austrinus Simon, 1910 & PW & tree \\
\hline & Monaeses fuscus Dippenaar-Schoeman, 1984 & PW & tree \\
\hline & Monaeses gibbus Dippenaar-Schoeman 1984 & PW & tree \\
\hline & Monaeses paradoxus (Lucas, 1846) & PW & tree \\
\hline
\end{tabular}


Table 2 continued . .

\begin{tabular}{|c|c|c|c|}
\hline FAMILY & SPECIES & GUILD & FIELD TYPE \\
\hline & Monaeses pustulosus Pavesi, 1895 & PW & tree \\
\hline & Monaeses quadrituberculatus Lawrence, 1927 & PW & tree \\
\hline & Oxytate argenteooculata (Simon, 1886) & PW & tree \\
\hline & Ozyptila sp. 1 & PW & soil \\
\hline & Pactactes trimaculatus Simon, 1895 & PW & soil \\
\hline & Pherecydes tuberculatus O.P.-Cambridge, 1883 & PW & tree \\
\hline & Pherecydes sp. 2 & PW & tree \\
\hline & Runcinia aethiops (Simon, 1901) & PW & grass \\
\hline & Runcinia affinis Simon, 1897 & PW & grass \\
\hline & Runcinia erythrina Jézéquel, 1964 & PW & grass \\
\hline & Runcinia flavida (Simon, 1881) & PW & grass \\
\hline & Stiphropus affinis Lessert, 1923 & PW & soil \\
\hline & Synema decens (Karsch, 1878) & PW & vegetation \\
\hline & Synema diana (Audouin, 1826) & PW & vegetation \\
\hline & Synema imitator (Pavesi, 1883) & PW & vegetation \\
\hline & Synema nigrotibiale Lessert, 1919 & PW & vegetation \\
\hline & Thomisops sulcatus Simon, 1895 & PW & vegetation \\
\hline & Thomisus blandus Karsch, 1880 & PW & grass \\
\hline & Thomisus citrinellus Simon, 1875 & PW & grass \\
\hline & Thomisus congoensis Comellini, 1957 & PW & grass \\
\hline & Thomisus granulatus Karsch, 1880 & PW & grass \\
\hline & Thomisus kalaharinus Lawrence, 1936 & PW & grass \\
\hline & Thomisus scrupeus (Simon, 1886) & PW & grass \\
\hline & Thomisus stenningi Pocock, 1900 & PW & grass \\
\hline & Tmarus africanus Lessert, 1919 & PW & grass \\
\hline & Tmarus cameliformis Millot, 1942 & PW & grass \\
\hline & Tmarus cancellatus Thorell, 1899 & PW & grass \\
\hline & Tmarus comellini Garcia-Neto, 1989 & PW & grass \\
\hline & Tmarus foliatus Lessert, 1928 & PW & grass \\
\hline & Tmarus Iongicaudatus Millot, 1941 & PW & grass \\
\hline & Tmarus sp. 7 & PW & grass \\
\hline & Xysticus fagei Lessert, 1919 & GW & soil \\
\hline \multirow[t]{4}{*}{ Uloboridae } & Miagrammopes longicaudus O.P.-Cambridge, 1882 & OWB & vegetation \\
\hline & Uloborus plumipes Lucas, 1846 & OWB & vegetation \\
\hline & Uloborus sp. 2 & OWB & vegetation \\
\hline & Uloborus sp. 3 & OWB & vegetation \\
\hline \multirow[t]{7}{*}{ Zodariidae } & Capheris decorata Simon, 1904 & GW & soil \\
\hline & Chariobas cylindraceus Simon, 1893 & GW & soil \\
\hline & Cydrela sp. 1 & GW & soil \\
\hline & Diores auricula Tucker, 1920 & GW & soil \\
\hline & Diores sp. 2 & GW & soil \\
\hline & Psammoduon sp. 1 & GW & soil \\
\hline & Ranops sp. 1 & GW & soil \\
\hline
\end{tabular}

other surveys undertaken in the Savanna Biome. In the Makalali Private Game Reserve, 4832 spiders were caught over a year represented by 38 families and 268 species (Whitmore et al. 2001); at Lajuma in the Western Soutpansberg 127 species were caught that represented 46 families (Foord et al. 2002); on Sovenga Hill in the Polokwane district 793 spiders were caught representing 29 families and 76 species (Modiba et al. 2005). In the Kruger National Park, 152 species from 40 families were recorded (Dippenaar-Schoeman \& Leroy 2003). The survey of the Ndumo Game Reserve reported the highest spider diversity from any protected area in South Africa so far with 46 families and 431 species (Haddad et al. 2006).

The most diverse families collected at the PNR were the Thomisidae (42 spp.), representing $15.3 \%$ of the total number of species sampled, followed by the Araneidae (39 spp.; 14.2\%) and the Salticidae (29 spp.; 10.5\%) (see Table 1). Sixteen families were represented by a single species only. At Roodeplaat Dam Nature Reserve and Lajuma, the Thomisidae were also the most diverse with 25 and 15 species respectively, while at Ndumu Nature Reserve, the Salticidae were the most diverse with 82 species. The total spider diversity (275 spp.) represents $13.75 \%$ of the species of South African spiders (2 000 spp.), presently protected in the Polokwane Nature Reserve.

The vast majority of the spiders (191 spp.) collected were wanderers $(69.5 \%)$, with web builders (84 spp.) contributing $30.5 \%$ (see Table 2). This follows the reported pattern for species of the Savanna Biome. At Ndumo Game Reserve, 75.9\% were wanderers, with web builders contributing $24.1 \%$ of the species (Haddad et al. 2006). At Makalali Private Game Reserve, the most abundant spiders collected were also wanderers (59\%), with web builders contributing $41 \%$ (Whitmore et al. 2002), while on Sovenga Hill, $64.9 \%$ of species were wanderers while the web builders only contributed $35.1 \%$ (Modiba et al. 2005). Similar results were found in the Western Soutpansberg with $64 \%$ of collected species being wanderers and $36 \%$ web builders (Foord et al. 2002).

Most of the Mygalomorphae spiders are protected due to the pet trade and it is important that species are protected in reserves. According to Dippenaar-Schoeman (2002b), there are 14 species of Mygalomorphae occurring in the Limpopo 
TABLE 3

Checklist of the mygalomorphs of the Polokwane Nature Reserve, Limpopo Province, South Africa

\begin{tabular}{ll}
\hline FAMILY & SPECIES \\
\hline Cyrtaucheniidae & Ancylotrypa brevipalpis (Hewitt, 1916) \\
Idiopidae & Idiops sp. \\
& Ctenolophus fernoulheti Hewitt, 1913 \\
& Segregara monticola (Hewitt, 1916) \\
Nemesiidae & Hermacha mazoena Hewitt, 1915 \\
Theraphosidae & Augacephalus junodi (Simon, 1904) \\
& Brachionopus pretoriae Purcell, 1904 \\
& Harpactira sp. \\
& Harpactirella sp. \\
\hline
\end{tabular}

Province. Only one of the previously recorded species (i.e. Augacephalus junodi (Simon, 1904)) was encountered during the study period. However, eight additional mygalomorph species (see Table 3) have been collected: Ancylotrypa brevipalpis (Hewitt, 1916), Brachionopus pretoriae Purcell, 1904, Ctenolophus fenoulheti Hewitt, 1913, Segregara monticola (Hewitt, 1916), Hermacha mazoena Hewitt, 1915, Idiops sp., Harpactira sp. and Harpactirella sp. All of the above-mentioned species are burrow dwelling. These species are all new geographical records for the province.

\section{CONCLUSION}

Considering the duration of the current study (one year) and the large number of specimens caught (13 821), it may be expected that the study provides a good representation of families occurring in the Polokwane Nature Reserve and that an extension of the study will probably increase the number of genera and species, but will probably not increase the number of families significantly. All the reported families and species are new geographical records for the Polokwane Nature Reserve.

The large number of species (97) that could not be identified emphasises the taxonomic impediment of invertebrates in South Africa. During this study, nine possible new species were collected, and several genera were collected in South Africa for the first time. Additionally, the study provided new information on the distribution of all species concerned and provides material that can be used in future taxonomic work. This highlights the important contribution that the SANSA initiative makes to a better knowledge of the diversity of arachnids in South Africa, especially in conserved areas.

\section{ACKNOWLEDGEMENTS}

We thank the NRF (Thuthuka programme) and the University of Limpopo (Research Development and Administration) for financial aid that supported this work; the Polokwane Municipality for allowing the study on their property; the Rotary Club (Polokwane) for allowing two of the authors to stay at the Education Centre for the duration of field work and for supplying additional accommodation together with Wood Worx; Die Drift Instant Lawn for sponsorship of a bicycle trailer and Saloojee Cycle Works for discount on bicycle repairs. Additionally we thank the University of Limpopo for provision of facilities and equipment; Ms B.P. Jordaan (Department of Biodiversity) for spending a lot of time assisting and organising to accommodate our needs in order to successfully complete the study; Ms A. van den Berg (ARC Plant Protection Research Institute) for her assistance with the family identification of specimens; and Mr C. Haddad (University of the Free State) for the identification of the Corinnidae specimens.

\section{REFERENCES}

Dippenaar-Schoeman,A.S.2002a.StatusofSouth AfricanArachnida fauna. Proceedings of the symposium on the status of South African species organised by the Endangered Wildlife Trust (EWT) of South Africa. Rosebank. 4 - 7 September 2002.

Dippenaar-Schoeman, A.S. 2002b. Baboon and trapdoor spiders of Southern Africa: an identification manual. Plant Protection Research Institute Handbook no. 13. Pretoria, Agricultural Research Council.

Dippenaar-Schoeman, A.S. \& Craemer, C. 2000. The South African National Survey of Arachnida (SANSA). Plant Protection News 56: 11-12.

Dippenaar-Schoeman, A.S. \& Jocqué, R. 1997. African spiders, an identification manual. Plant Protection Research Institute Handbook no. 9. Pretoria, Agricultural Research Council.

Dippenaar-Schoeman, A.S. \& Leroy, A. 2003. A check list of the spiders of the Kruger National Park, South Africa (Arachnida: Araneae). Koedoe 46: 91-100.

Dippenaar-Schoeman, A.S., Van den Berg, A. \& Van den Berg, A.M. 1989. Species composition and relative seasonal abundance of spiders from the field and tree layers of the Roodeplaat Dam Nature Reserve. Koedoe 32: 25-38.

Foord, S.H., Dippenaar-Schoeman, A.S. \& Van der Merwe, M. 2002. A checklist of the spider fauna of the Western Soutpansberg, South Africa (Arachnida: Araneae). Koedoe 45: 35-43.

Haddad, C.R., Dippenaar-Schoeman, A.S. \& Wesolowska, W. 2006. A checklist of the non-acarine arachnids (Chelicerate: Arachnida) of the Ndumo Game Reserve, Maputaland, South Africa. Koedoe 49: 1-22.

Low, A.B. \& Rebelo, A.G. (eds). 1996. Vegetation of South Africa, Lesotho and Swaziland. Pretoria, Department of Environmental Affairs and Tourism.

Myers, N. 1990. The biodiversity challenge: expanded hot-spot analysis. The Environmentalist 10: 243-255.

Modiba, M.A., Dippenaar, S.M. \& Dippenaar-Schoeman, A.S. 2005. A checklist of spiders from Sovenga Hill, an inselberg in the Savanna Biome, Limpopo Province, South Africa (Arachnida: Araneae). Koedoe 48: 109-115.

Mucina, L. \& Rutherford, M.C. (eds). 2006. The vegetation of South Africa, Lesotho and Swaziland. Strelitzia 19. Pretoria, South African Biodiversity Institute.

Mucina, L., Rutherford, M.C. \& Powrie, L.W. 2005. Vegetation map of South Africa, Lesotho and Swaziland. South Africa National Biodiversity Institute (SANBI), Kirstenbosch Research Centre.

Whitmore, C., Slotow, R., Crouch, T.E. \& Dippenaar-Schoeman, A.S. 2001. Checklist of spiders (Araneae) from a Savanna ecosystem, Northern Province, South Africa: including a new family record. Durban Museum Novitates 26: 10-19.

Whitmore, C., Slotow, R., Crouch, T.E. \& Dippenaar-Schoeman, A.S. 2002. Diversity of spiders (Araneae) in a Savanna Reserve, Northern Province, South Africa. Journal of Arachnology 30: 344-356. 